\title{
Should we regionalize the management of pulmonary embolism?
}

\author{
Marc Carrier MD, Philip S. Wells MD MSc
}

$\mathrm{V}$ enous thromboembolism, which manifests as either deep vein thrombosis or pulmonary embolism, is one of the most common cardiovascular disorders in industrialized countries, affecting about $5 \%$ of people in their lifetime. ${ }^{1}$ Pulmonary embolisms are highly fatal, and in $22 \%$ of cases, they are not diagnosed until after they cause death. ${ }^{2}$ If untreated, pulmonary embolism has a high mortality and accounts for $5 \%-10 \%$ of all in-hospital deaths. ${ }^{2,3}$ Registry data shows an overall 3-month mortality of $17.4 \% .{ }^{4}$ Of these deaths, $45 \%$ were ascribed to pulmonary embolism and $75 \%$ occurred during the initial hospital admission for pulmonary embolism. There is room for considerable improvement in the management of pulmonary embolism, but it remains unknown whether preventive measures, diagnosis, treatment modalities, or physician or patient education should be the focus. Research that may help elucidate the problem may help to create a solution.

In this issue, Aujesky and colleagues report that pulmonary embolism treated in hospitals with high case volume is associated with reduced short-term mortality. ${ }^{1}$ In 1979 , Luft and colleagues reported that hospital volume and mortality were inversely related. They found that mortality of open-heart surgery, vascular surgery, transurethral resection of the prostate and coronary bypass decreased with an increasing number of operations. ${ }^{5}$ This finding has led to the dilemma about whether to regionalize complex cases to high-volume medical centres. The association between case volume and outcome has been documented numerous times, fueling the debate. ${ }^{5}$ In some settings, regionalizing traumatology and perinatalogy has been successful and has shown benefits. ${ }^{6} \mathrm{~A}$ systematic review by Halm and colleagues that included 27 trials showed that $7 \mathrm{I} \%$ of all studies of hospital volume and $69 \%$ of studies of physician volume reported a statistically significant association between high volume and improved patient outcome. Of the included studies, none reported a statistically significant association between high volume and worse outcome. ${ }^{7}$ Recent studies have suggested a correlation between hospital volume and patient health and function after surgery. ${ }^{8,9}$ Similar results have been reported among patients receiving mechanical ventilation ${ }^{10}$ but not among patients with pneumonia. ${ }^{11}$

Two principal hypotheses have been proposed to explain the relation between volume and outcome. First, health care
Important elements of a pulmonary embolism program

- Pulmonary embolism should be diagnosed based on proven algorithms that, when necessary, include prompt diagnostic imaging.

- Continuous hospital coverage should be provided by physicians experienced in diagnosing and managing pulmonary embolism.

- Anticoagulation control can be improved when care is centralized at an anticoagulation clinic.

- Programs should strive to coordinate care, adopt institutionand region-wide guidelines, and develop and implement appropriate quality-of-care programs.

providers may develop more effective skills if they care for more patients or perform a procedure more often. Second, providers who achieve better outcomes may be referred more patients and thus accrue larger case volumes (selection referral). ${ }^{7}$

The study by Aujesky and colleagues suggests that the regionalized management of pulmonary embolism at highvolume centres could improve patient outcomes. Regionalized thrombosis programs with appropriate leadership may increase the uptake of evidence that improves diagnosis and treatment. The following are important elements of a successful pulmonary embolism program. First, pulmonary embolism should be diagnosed following a proven diagnostic algorithm and include prompt diagnostic imaging (computed tomography, ventilation-perfusion scans, ultrasonography or pulmonary angiography), which may not be available in lowvolume settings. Evidence has proven the value of following diagnostic algorithms. ${ }^{12,13}$ Second, continuous hospital coverage should be provided by physicians experienced in the diagnosis and management of pulmonary embolism. Studies have shown that mortality is higher among patients admitted to hospital with a pulmonary embolism on weekends compared with weekdays. ${ }^{14}$ Continuous coverage may not be available in lower-volume settings. Third, appropriate anticoagulation control significantly reduces the risk of death from

From the Department of Medicine, Ottawa Hospital, Ottawa Health Research Institute and the University of Ottawa, Ottawa, Ont. 
a pulmonary embolism. Because major hemorrhage and poor control of international normalized ratio frequently occur among patients taking anticoagulants during the first month after discharge from hospital, ${ }^{15,16}$ anticoagulation control should be managed at a central anticoagulation clinic where the quality of control is improved compared with family practices. ${ }^{15}$ Finally, access to thrombolysis and intensive care monitoring should be available if required. High-volume providers may have better access to these services than lowvolume providers. Successful regionalized thrombosis programs need to ensure coordination of the care, adoption of institution- and region-wide guidelines, and development and implementation of appropriate quality-of-care programs.

Policy-makers need to take action in light of increasing evidence showing that, for certain diagnoses (perhaps including pulmonary embolism), care at higher-volume centres leads to better outcomes. However, the volume-outcome correlation hypothesis proposed by Aujesky and colleagues is imperfect. First, the identification of events in their study is problematic. Aujesky and colleagues used International Classification of Diseases (ninth revision, clinical modification [ICD-9CM]) codes to identify pulmonary embolism without any chart review. For venus thromboembolism coding, the specificity is $74 \%$ and the positive predictive value is $62 \%{ }^{17}$ Second, if case volume is associated with outcome, it is difficult to explain why mortality was significantly lower in low-volume hospitals (I0-I9 cases per year) compared with high-volume hospitals (20-4I cases per year). Third, Aujusky and colleagues found that only $4.3 \%$ of all pulmonary embolism patients receive care in very-low-volume hospitals (< Io cases per year) and that the absolute 30-day mortality difference was only 3.4\%. Given this, it is unlikely that mechanisms to provide pulmonary-embolism care in higher-volume centres would have a large effect on overall population mortality. Furthermore, these differences in mortality are not necessarily all related to pulmonary embolism. In fact, it is likely that less than half are related pulmonary embolisms and, instead, may be related to other processes of care, suggesting that better care of the pulmonary embolism would have no effect on outcome. The prognostic model used by Aujesky and colleagues predicts mortality among patients with pulmonary embolism, not mortality due to pulmonary embolism.

The volume-outcome correlation hypothesis is imperfect, and a wide variation of outcomes may be observed among high-volume providers. ${ }^{7}$ Differences in case mix and severity, as well as in processes of care between high- and low-volume providers may explain part of the observed relation; however, Aujesky and colleagues were unable to account for mortality differences owing to process of care. The most striking difference in mortality between high- and low-volume providers has been reported for high-risk procedures and conditions (e.g., pancreatic cancer, abdominal aortic aneurysm). ${ }^{7}$ The magnitude of the relation between volume and outcome is significantly reduced for more common conditions (e.g., pneumonia). Furthermore, a wide variation of outcomes may be observed among high-volume providers. For example, in a detailed analysis of 159 surgeons who performed a high or very high volume of radical prostatectomy, Begg and col- leagues reported a wider variation among surgeons than predicted based on chance or case-mix variations. ${ }^{9}$ This suggests that volume may not be a perfect surrogate marker for quality of care and that we need to carefully interpret the clinical significance of these volume-outcome correlations. Identifying the relative contribution of the different factors involved in the processes of care (e.g., composition of medical teams, treatment protocols, resource availability) would be ideal. However, this would be a substantial undertaking and such efforts should be focused where results are most likely to be fruitful. Furthermore, the relative contribution of the hospital- and physician-volume are largely unknown because very few studies have evaluated both types of volume simultaneously. Therefore, a selective-referral policy to a high-volume provider (hospital or physician) may not achieve the desired effect - a low-volume physician in a high-volume hospital may have worse outcomes than an average-volume physician in an average-volume hospital. ${ }^{7}$ Further research should be performed on the contribution of process of care and hospital volume and physician volume, as well as on the longitudinal trend of volume-outcome correlations for patients with pulmonary embolism.

The ultimate goal is to develop safety- and quality-assurance programs that monitor and improve care in all hospitals in order to provide equal care to all patients. We are not convinced that referring patients with pulmonary embolism to highervolume centres would significantly reduce mortality. It is clear that following diagnostic algorithms and treatment guidelines is a minimal requirement and that a starting point may be to compare the diagnostic and treatment processes in high- and low-volume centres. Increasing the proportion of care provided in high-volume centres by selective referral is worth exploring. Lessons learned from successful regional care programs, including traumatology and perinatology, may be helpful. However, we need to consider access to care for patients in rural areas, avoidance of duplication of services, and financial and resource constraints for high-volume providers in any study that evaluates regionalization of treatment to higher-volume centres.

Competing interests: None declared for Marc Carrier. Philip Wells has received speaker fees from Organon, BioMérieux and Sanofi Aventis.

Contributors: Both of the authors contributed to the conception and design of the manuscript, drafted the article and revised it critically for important intellectual content. Both of the authors approved the final version for publication.

Acknowledgements: Dr. Wells is the recipient of a Canada Research Chair in Thromboembolic Diseases.

\section{REFERENCES}

I. Aujesky D, Mor MK, Geng M, et al. Hospital volume and patients outcomes in pulmonary embolism. CMAJ 2007;178:27-33.

2. Heit JA, O'Fallon WM, Petterson TM, et al. Relative impact of risk factors for deep vein thrombosis and pulmonary embolism: a population-based study. Arch Intern Med 2002;162:1245-8.

3. Anderson FA Jr, Wheeler HB, Goldberg RJ, et al. A population-based perspective of the hospital incidence and case-fatality rates of deep vein thrombosis and pulmonary embolism. The Worcester study. Arch Intern Med I991;151:933-8.

4. Goldhaber SZ, Visani L, De Rosa M. Acute pulmonary embolism: clinical outcomes in the International Cooperative Pulmonary Embolism Registry (ICOPER). Lancet $1999 ; 353: 1386-9$. 
5. Luft HS, Bunker JP, Enthoven AC. Should operations be regionalized? The empirical relation between surgical volume and mortality. N Engl J Med I979;301:1364-9.

6. Nathens $A B$, Jurkovich GJ, Cummings $P$, et al. The effect of organized systems of trauma care on motor vehicle crash mortality. JAMA 2000;283:1990-4.

7. Halm EA, Lee C, Chassin MR. Is volume related to outcome in health care? A systematic review and methodologic critique of the literature. Ann Intern Med 2002;137:5II-20.

8. Birkmeyer JD, Siewers AE, Finlayson EVA, et al. Hospital volume and surgical mortality in the United States. N Engl J Med 2002;346:1128-37.

9. Begg CB, Riedel ER, Bach PB, et al. Variations in morbidity after radical prostatectomy. NEngl J Med 2002;346:1138-44.

Io. Kahn JM, Goss CH, Heagertly PJ, et al. Hospital volume and the outcome of mechanical ventilation. N Engl J Med 2006;355:4I-50.

II. Lindenauer PK, Behal R, Murray CK, et al. Volume, quantity of care and outcome in pneumonia. Ann Intern Med 2006; I44:262-9.

I2. Wells PS, Anderson DR, Rodger MA, et al. Excluding pulmonary embolism at the bedside without diagnostic imaging: management of patients with suspected pulmonary embolism presenting to the emergency department by using a simple clinical model and D-dimer. Ann Intern Med 2001;135:98-107.
I3. Roy PM, Meyer G, Vielle B, et al. Appropriateness of diagnostic management and outcomes of suspected pulmonary embolism. Ann Intern Med 2006;I44:157-64.

I4. Bell CM, Redelmeier DA. Mortality among patients admitted to hospitals on weekends as compared with weekdays. N Engl J Med 2001;345:663-8.

I5. Oake N, Fergusson DA, Forster AJ, et al. Frequency of adverse events in patients with poor anticoagulation: a meta-analysis. $C M A J$ 2007;176:1589-94.

I6. van Walraven C, Oake N, Wells PS, et al. Burden of potentially avoidable anticoagulant-associated hemorrhagic and thromboembolic events in the elderly. Chest 2007;131:1508-I5.

I7. Arnason T, Wells PS, van Walraven C, et al. Accuracy of coding for possible warfarin complications in hospital discharge abstracts. Thromb Res 2006;118:253-62.

Correspondence to: Dr. Philip Wells, Suite F6-49, 1053 Carling Ave., Ottawa Hospital Civic Campus, Ottawa ON $\mathrm{KIY}_{4}$ E9; fax61376I-535I; pwells@ohri.ca

\section{BMA - AMA - CMA International Conference on Doctors' Health}

\section{Doctors' Health Matters - Finding The Balance}

\section{Monday 17 - Wednesday 19 November 2008}

Venue: British Medical Association, BMA House, Tavistock Square, London, WC1H 9JP, United Kingdom

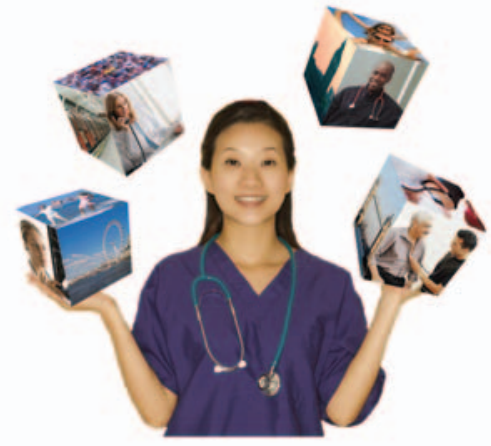

\section{ABSTRACTS INVITED}

Doctors' good health is important to all of us, both as doctors and as patients. This conference seeks to promote a healthier culture of medicine for doctors, and to reduce the stigma associated with ill health in doctors.

The conference will:

- Provide a forum for practitioners and researchers to present recent findings, innovative treatments and educational programmes in the area of doctors' health

- Provide an environment for networking, exchange of information and leisure activity focused on staying healthy

The Call for Papers is now open and submissions can be accepted online at www.bma.org.uk/doctorshealthmatters

The closing date for receipt of abstracts is Thursday 7 February 2008.

Put the date in your diary now and check www.bma.org.uk/doctorshealthmatters for further details.

For further information on the conference and the call for papers, please contact BMA Conferences on +44 (0)20 7383 6605/6137 or email doctorshealthmatters@bma.org.uk

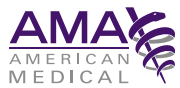

ASSOCIATION CANADIAN

MÉDICALE \$ MEDICAL

CANADIENNE \& ASSOCIATION 